\title{
Intrasellar Region
}

National Cancer Institute

\section{Source}

National Cancer Institute. Intrasellar Region. NCI Thesaurus. Code C155779.

The area inside the sella turcica. 